\title{
La influencia de las nuevas tecnologías de la información y comunicación en la educación superior.
}

\section{The influence of new information and communication technologies in higher education.}

Verónica Oña Gamboa. ${ }^{1}$, Danilo Miniguano Miniguano. ${ }^{2}$, Gregorio Aurelio Camacho Reina. $^{3}$ \& Darwin Andrés Naranjo Villota. ${ }^{4}$

\begin{abstract}
.
DOI: https://doi.org/10.33262/cienciadigital.v3i4.937

The advancement of technology is growing by leaps and bounds so it is necessary to maintain a constant preparation and interact with innovative teaching methods applied to education so as not to fall into digital illiteracy, therefore, the demand for Internet connectivity is increasingly high for what today's youth see as a necessity in the teaching-learning process based on the current educational revolution.

Information and Communication Technologies (ICT) play a fundamental role in research, so it is necessary to prioritize investment in order to improve the quality of teaching in the classroom, which is why governments must commit a greater investment in education, betting on educational scholarships for visionary young university students betting on the future of the nation.

The future is not far and is projected to a digitalized society with a high technological demand in search of knowledge and the integration of ICT.
\end{abstract}

Keywords: ICT, education, technology.

\footnotetext{
1 Universidad Técnica de Ambato, Ambato, Ecuador, verona90957@gmail.com

${ }^{2}$ Instituto Superior Tecnológico Sucre, Quito, Ecuador, dminiguano@tecnologicosucre.edu.ec

${ }^{3}$ Universidad Técnica Estatal de Quevedo, Quevedo, Ecuador, gregorio.camacho@uteq.edu.ec

${ }^{4}$ Unidad Educativa Carlos Martínez Acosta, Ecuador, darwin.naranjo@educacion.gob.ec
} 


\section{Resumen.}

El avance de la tecnología crece a pasos agigantados por lo que es necesario mantener una constante preparación e interactuar con métodos de enseñanza innovadores aplicados a la educación para no caer en el analfabetismo digital, por ello, la demanda de conectividad a Internet cada vez es más alto por lo que la juventud actual lo ve como una necesidad en el proceso de enseñanza- aprendizaje en función de la revolución educativa actual.

Las Tecnologías de la Información y Comunicación (TIC) cumplen un papel fundamental en la investigación por lo que se requiere priorizar la inversión con la finalidad de mejorar la calidad de enseñanza en las aulas, es por ello que los gobiernos deben comprometer una mayor inversión en la educación, apostando a becas educativas para los jóvenes universitarios visionarios apostando al futuro de la nación.

El futuro no está lejos y se proyecta a una sociedad digitalizada con una alta demanda tecnológica en busca del conocimiento y la integración de las TIC.

Palabras claves: TIC, educación, tecnología.

\section{Introducción.}

Los cambios sustanciales en la educación que han transformado todo el entorno dejando atrás la vieja escuela, encaminada a cambios constantes que involucran las TIC y el impacto en la educación (Illich, 1974).

El desarrollo tecnológico y el crecimiento agigantado de las TIC cambiaron la visión general de la sociedad, buscando nuevos caminos y técnicas que permitan adaptarse a la revolución tecnológica del siglo XXI y el impacto que causa en la educación (Pérez-Fabara, 2017).

El avance tecnológico, conjuntamente con el desarrollo de las TIC ha modificado el entorno global de la sociedad, obligando a realizar cambios para adaptarse a una revolución que crece desmedidamente causando un impacto directo en la educación (Moya, 2016).

Muchos autores hablan del crecimiento tecnológico y las consecuencias que produce, por lo que es importante fortalecer el trabajo en todos los campos de la enseñanza, logrando cambios sustanciales en los métodos y técnicas de enseñanza encaminados a buscar cambios sustanciales en la sociedad y la adaptación a los mismos (Zangara, 2008).

Estos cambios trascendentales involucran conocimientos nuevos y mejor preparación en todos los campos del conocimiento fortaleciendo la investigación en las universidades en busca del conocimiento (Moya E. J., 2016).

La evolución de las TIC requiere esfuerzos grandes con visión a cambios trascendentales encaminados a un desarrollo paralelo de la ciencia y la tecnología. 
La evolución tecnológica y el crecimiento económico nos encaminan a cambiar la perspectiva general de las carreras buscando innovar las técnicas y métodos de enseñanza aprendizaje que permitan cambios sustanciales en las carreras (Vessuri, 1993).

\section{Metodologia.}

\section{Generalidades}

"Nunca antes en la historia el bienestar de las naciones ha estado tan estrechamente vinculado a la calidad y el alcance de sus sistemas e instituciones de enseñanza superior" (Conferencia Mundial sobre la Educación Superior, junio de 2003).

La UNESCO fomenta la innovación con el fin de satisfacer las necesidades de la enseñanza y del mercado laboral, y examina de qué manera se pueden aumentar las oportunidades educativas de los jóvenes y los grupos desfavorecidos.

El intercambio cultural y homologación de diplomas son políticas que contribuyen al crecimiento y fortalecimiento investigativo de las instituciones que sobrepasa el límite de las fronteras (UNESCO, 2016).

\section{La tecnología como base del crecimiento tecnológico en la educación}

\section{Acceso al Internet en el Ecuador}

El internet juega un papel preponderante para la aplicación de las TIC en la educación por lo que es muy importante la inversión del estado para el fortalecimiento buscando que sea política de estado el acceso a internet como parte de una sociedad tecnológica encaminada al desarrollo.

Según el (INEC, 2013) el 28,3\% de los hogares a nivel nacional tienen acceso a internet observando un crecimiento sustancial a referencia del año 2010. Este crecimiento se ve reflejado en área urbana con 20,3 puntos y en el área rural 16,5 puntos como se puede ver en la figura 1.

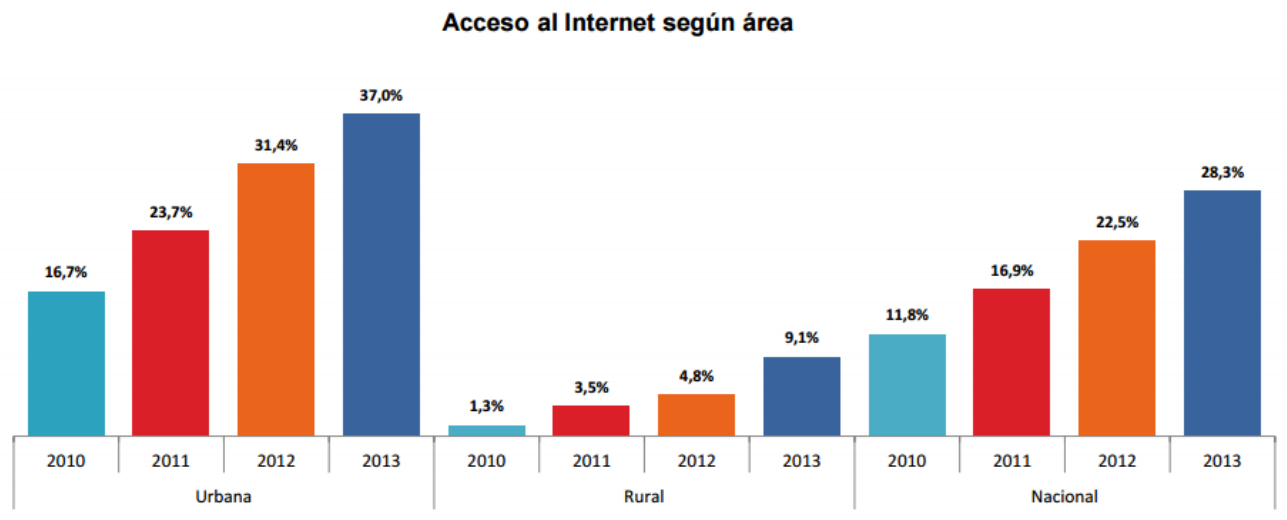

Figura 1. Acceso a internet

Fuente: Encuesta Nacional de Empleo, Desempleo y Subempleo - ENEMDU (2010-2013) 


\section{Hogares que tienen acceso a internet a nivel Nacional}

Un hogar sin internet es como un computador sin energía, en la actualidad es fundamental el servicio ya que todo está encaminado al uso de las TIC como herramientas de apoyo para la educación.

En la figura 2, se puede ver que el $28,3 \%$ de los hogares tiene acceso a Internet, de ellos el $43,7 \%$ accede a través de modem o teléfono, 9,8 puntos menos que en 2012 (INEC, 2013).

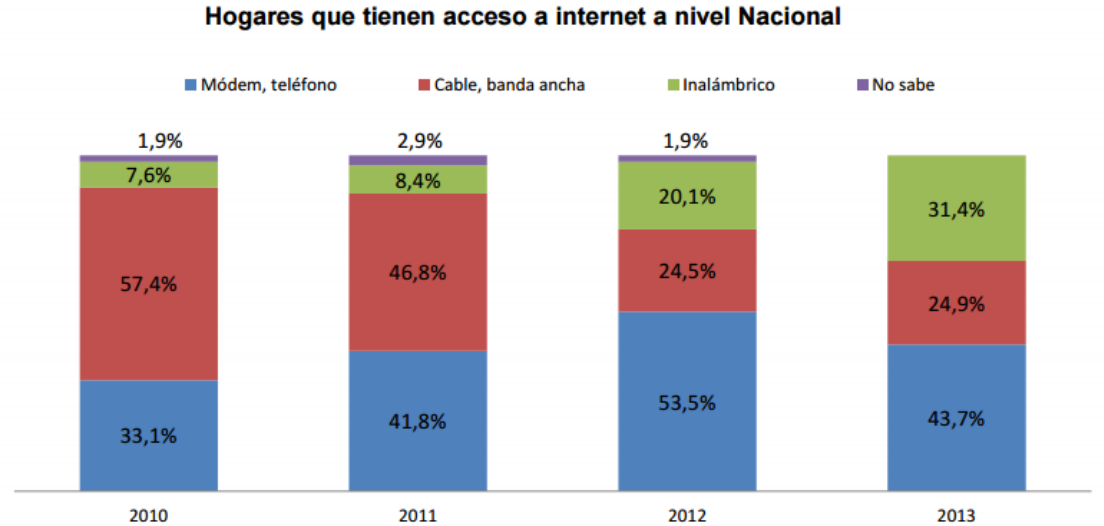

¿Tiene este HOGAR: Módem/Teléfono? ¿Cable/Banda Ancha? Inalámbrico? Ninguno? ENEMDU 2010 - 2012 ¿A través de línea telefónica? A través de cable? ¿A través de medio inalámbrico BAM-3G? ENEMDU 2013

Figura 2. Hogares con Acceso a internet

Fuente: Encuesta Nacional de Empleo, Desempleo y Subempleo - ENEMDU (2010-2013)

\section{Porcentaje de personas que utilizan computadora por grupos de edad a nivel nacional.}

$\mathrm{El}$ analfabetismo digital es uno de los principales factores que impiden un desarrollo paralelo de la sociedad por lo que es importante buscar crear entornos que permitan capacitar a la población en el uso de la tecnología y la importancia que tienen en la educación (Ver figura $3)$.

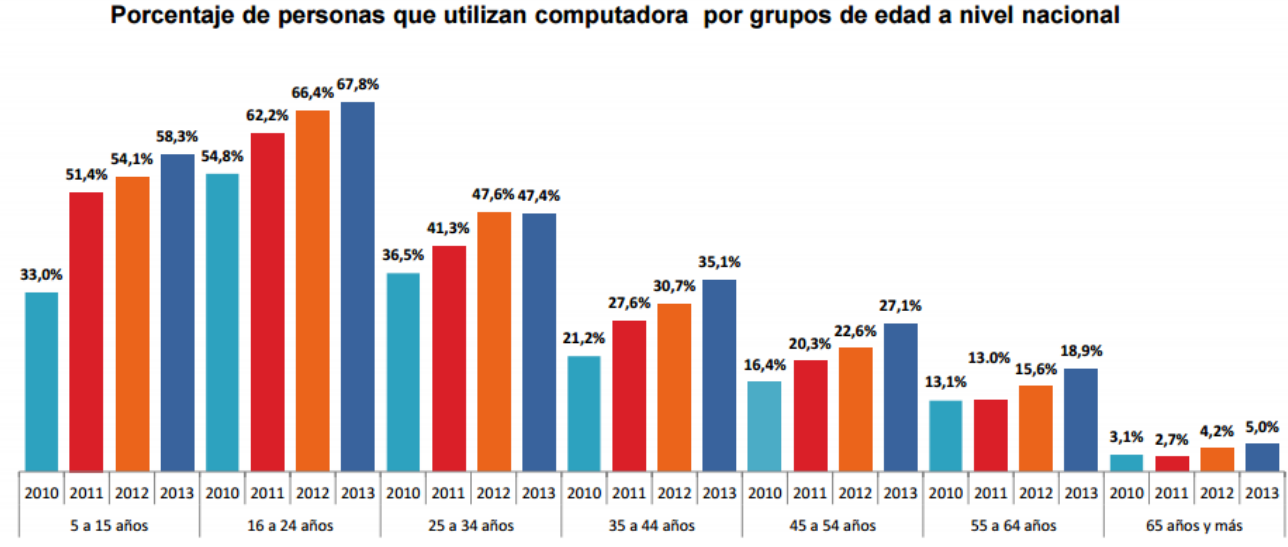

Figura 3. Personas que utilizan computadora

Fuente: Encuesta Nacional de Empleo, Desempleo y Subempleo - ENEMDU (2010-2013) 
El grupo etario con mayor número de personas que utilizaron computadora es el que está entre 16 a 24 con el 67,8\%, seguidos de 5 a 15 años con 58,3\% (INEC, 2013).

De las estadísticas los estudiantes de 18 a 25 años son estudiantes de nivel superior por lo que es una buena muestra que garantiza el crecimiento tecnológico que tienen los jóvenes universitarios.

\section{Porcentaje de personas que utilizan computadora por provincias en el 2013}

En la figura 4, se evidencia que la provincia con mayor número de personas que utilizan computadora es Pichincha con el 56,2\%, seguido por Azuay con el 54,3\%; mientras que Los Ríos es la provincia que menos usa con el 28,4\% (INEC, 2013).

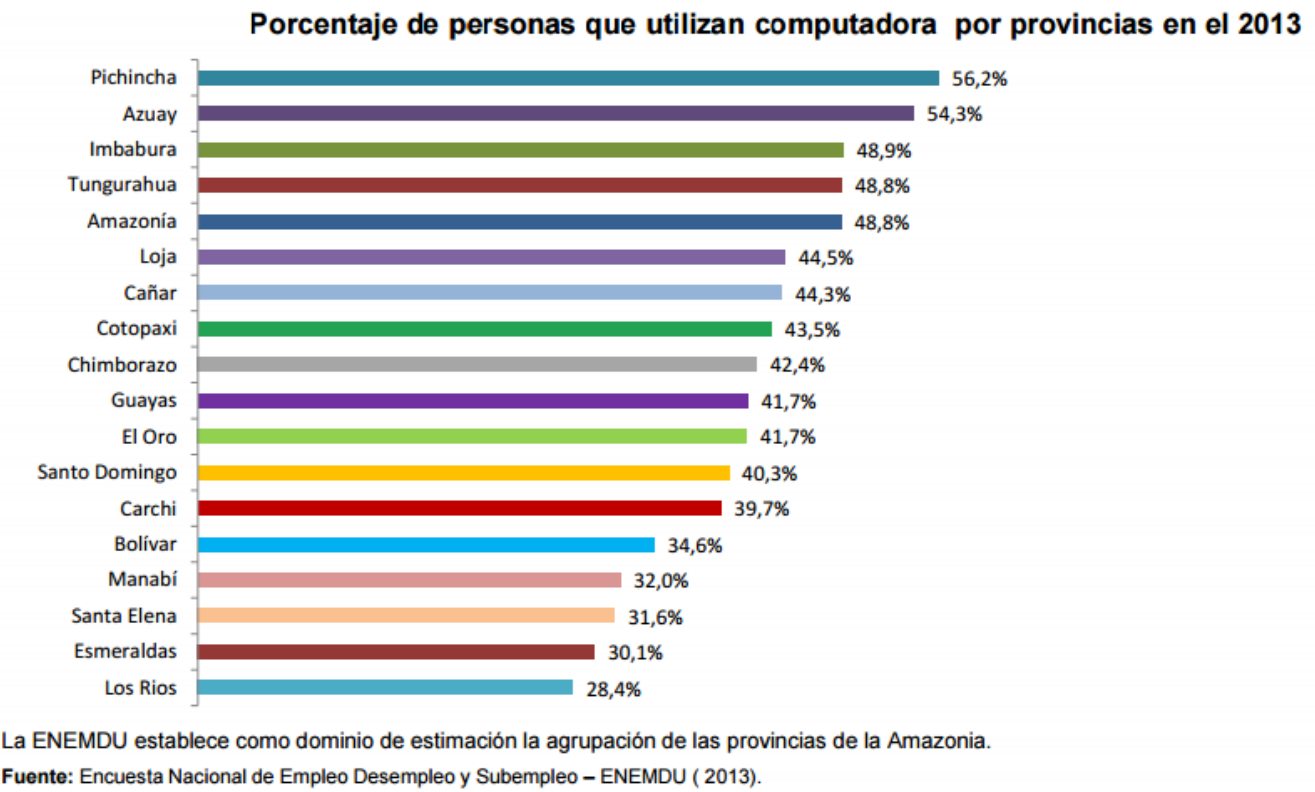

Figura 4. Personas que usan computadora por provincias

Fuente: Encuesta Nacional de Empleo, Desempleo y Subempleo - ENEMDU (2010-2013)

La región sierra tiene buenas cifras y la importancia de saber utilizar un computador, pero hay mucho por hacer ya que no es suficiente con aprender más bien es importante mantenerse caminar al ritmo del crecimiento tecnológico actual.

\section{Razones de uso de Internet por área}

Es necesario concientizar el uso adecuado de los recursos tecnológicos e internet en la juventud actual ya que en la mayoría de los casos existe un crecimiento desmedido de redes sociales y la difusión de información basura que poco o nada ayudan en los procesos de enseñanza-aprendizaje. 
En la figura 5, se puede ver que en el 2013, el 32,0\% de las personas usó Internet como fuente de información, mientras el 31,7\% lo utilizó como medio de educación y aprendizaje (INEC, 2013).

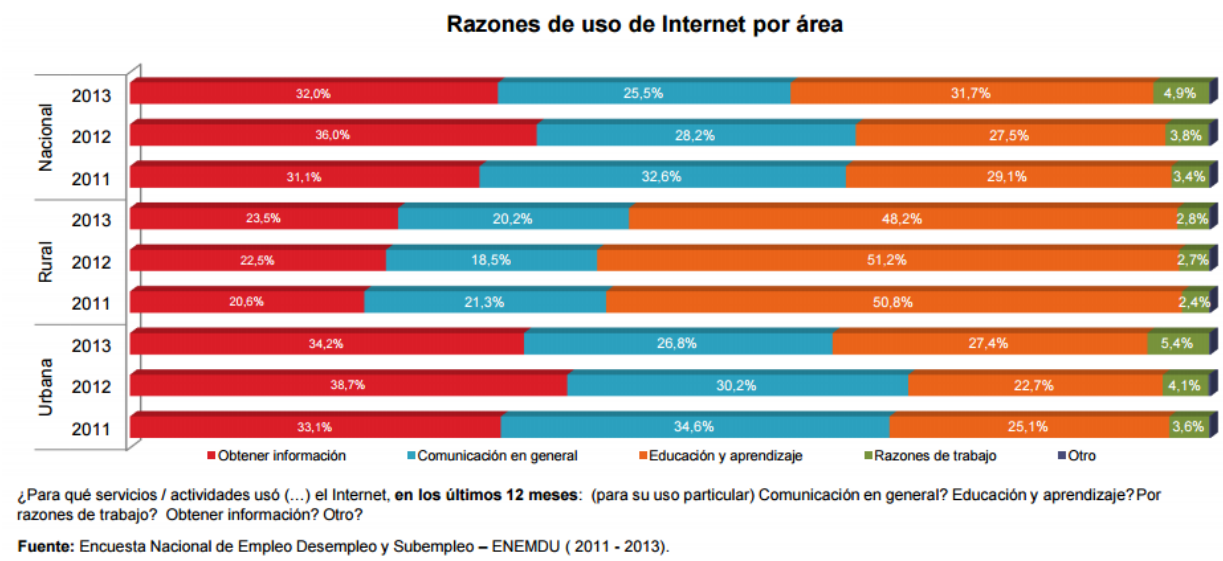

Figura 5. Razones de uso del internet

Fuente: Encuesta Nacional de Empleo, Desempleo y Subempleo - ENEMDU (2010-2013)

Es importante el porcentaje que utiliza como medio de educación y aprendizaje, pero se requiere mejorar estas cifras para garantizar el aprovechamiento de la tecnología en la educación superior.

\section{El Estado como pilar de la Educación superior}

La inversión en educación superior, la asignación del Producto Interno Bruto (PIB) para esa área, la entrega de la beca 10.000, son parte de los hitos que le permiten al Estado ecuatoriano identificar al 2014 como el año de la ciencia, la tecnología y la educación superior, informó la Senescyt.

"La inversión en educación superior en los últimos siete años del Gobierno fue de 9.445 millones de dólares, la asignación del Producto Interno Bruto (PIB) para esa área pasó del 0,72\% en el 2006 al 2.12\% en el 2014, lo que identifica al Ecuador como el país que más invierte en educación superior de toda la región, son los pilares del cambio el año pasado anunció la Secretaría de Educación Superior, Ciencia, Tecnología e Innovación (Senescyt)" (Andes, 2016).

El incremento de becas para estudios de tercer y cuarto nivel como política de estado ha logrado incentivar a la comunidad estudiantil, levantado el espíritu investigador que en la actualidad es necesario para fortalecer las bases de una sociedad en constante evolución.

La inserción de 861 Prometeos que colaboran en las instrucciones a nivel nacional es un aporte fundamental para el intercambio de conocimientos y el fortalecimiento de la educación con miras al futuro. 
Otra de las políticas que ayudan a desarrollo del conocimiento es la democratización del conocimiento que garantiza la igualdad de oportunidades y el libre acceso a una educación de calidad.

\section{Las universidades y su rol}

Las universidades deben enfocar sus esfuerzos en brindar el apoyo necesario al desarrollo de las TIC'S como herramienta fundamental de la educación en la actualidad, fortaleciendo su infraestructura tecnológica y la planta docente para generar competitividad en la comunidad estudiantil.

Es importante la inversión pública como privada que se da a la educación, pero aún hay mucho por hacer ya que es necesario un mayor aporte que garantice un desarrollo sostenido encaminado al crecimiento investigativo y la búsqueda del conocimiento.

\section{Los docentes y las NTIC}

El campo educativo actual requiere el apoyo de las TIC por lo que es importante la capacitación adecuada al personal docente, además de la incorporación de herramientas adecuadas para impartir el conocimiento.

Según (Cardoso, 2001), "Un medio puede definirse como cualquier forma de instrumento o equipamiento que se utiliza normalmente para transmitir información". En la actualidad los medios educacionales se consideran las computadoras, internet y herramientas multimedia.

Los componentes esenciales dentro del proceso educativo son: proyectores, pizarra electrónica, tabletas, equipos móviles, reproductores de medios digitales, etc., además existe una amplia gama de aplicaciones tales como plataformas digitales, wikis, blogs, YouTube, simuladores y más.

Es importante seleccionar adecuadamente las herramientas tecnológicas a compartir con la finalidad de mejorar el entorno de aprendizaje que se genera para el estudiante.

\section{Las NTIC en los procesos de enseñanza y de investigación}

Diversas aplicaciones están orientadas a facilitar los entornos pedagógicos, por lo que es importante seleccionar las herramientas y el material adecuado a utilizar en el aula.

Las TIC a menudo limita el proceso de enseñanza-aprendizaje ya que facilitan el entretenimiento de los alumnos con juegos, propaganda y links nada favorables para el desarrollo adecuado de la investigación. 


\section{Uso de las tics tradicionalistas}

Según la (UIT, 2015) el crecimiento de las TIC ha sido sustancial permitiendo un crecimiento socioeconómico en todos los ámbitos sociales como se ve en la figura 6 .

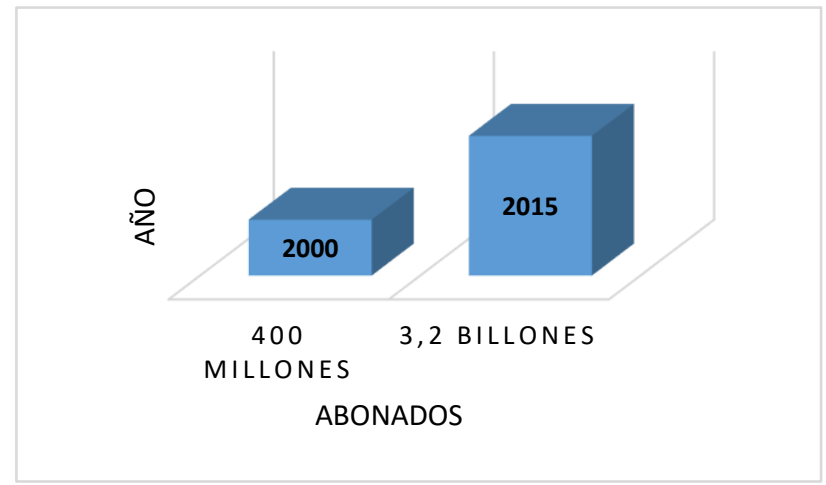

Figura 6. Conectividad de usuarios a internet en el mundo

Fuente: Encuesta Nacional de Empleo, Desempleo y Subempleo - ENEMDU (2010-2013)

\section{Uso de las TIC innovadores}

El avance de las TIC en países de Latinoamérica es considerable pero no es suficiente ya que se analiza, entre otras variables, el grado con el que la población está preparada para hacer uso de las infraestructuras y los contenidos digitales, por eso, dentro de las conclusiones del informe se destaca que los países no se deben enfocar en el desarrollo de las instalaciones, sino que también deben pensar en implementar diferentes estrategias encaminadas a crear condiciones para las competencias, la innovación, etc. (Colombia Digital, 2014).

\section{TIC futuristas}

"Las TIC desempeñarán un papel más significativo en la era posterior a 2015 y en el futuro cumplimiento de los objetivos de desarrollo sostenible a medida que el mundo evoluciona cada vez más rápido hacia una sociedad digital", declaró el Sr. Brahima Sanou, Director de la Oficina de Desarrollo de las Telecomunicaciones de la UIT. "Nuestra misión es conectar a todos y crear una sociedad de la información realmente integradora, para lo cual necesitamos disponer de datos y estadísticas de elevada calidad que nos permitan cuantificar el progreso" (UIT, 2015).

\section{Conclusiones}

- Entre 2000 y 2015 la penetración de Internet se ha multiplicado casi por siete, pasando de $6,5 \%$ al $43 \%$ de la población mundial, lo que evidencia que el uso de internet en los niños, jóvenes y adultos tienen un avance desmesurado e incontrolable. 
- En cuanto a la proporción de hogares con acceso a Internet, el avance tecnológico genero un aumento del $18 \%$ en el 2005 al $46 \%$ en el 2015 , lo cual afirma que cada vez más existe menos brecha digital en el uso del internet.

- Los datos de la UIT también indican que en el mundo en desarrollo sigue habiendo 4000 millones de personas sin acceso a Internet; y de los casi 1000 millones de personas que viven en países menos adelantados, 851 millones no utilizan Internet.

\section{Referencias bibliográficas.}

Andes. (Febrero de 2016). Obtenido de http://www.andes.info.ec/es/noticias/ecuadoridentifica-2014-ano-ciencia-tecnologia-educacion-superior.html

Cardoso, J. (Diciembre de 2001). América Learning \& Media. Obtenido de http://www.americalearningmedia.com/edicion-009/111-white-papers/687-tic-en-elaula-materiales-medios-y-tecnologia-educativa

Colombia Digital. (Abril de 2014). Ranking mundial sobre el uso de las TIC. Obtenido de https://colombiadigital.net/actualidad/noticias/item/6953-ranking-mundial-sobre-eluso-de-las-tic.html

Illich, I. (1974). La desescolarización. Barcelona: Barral. Obtenido de http://educrea.cl/laformacion-docente-para-el-uso-de-las-tecnologias-de-la-informacion-y-de-lacomunicacion/

INEC. (2013). Ecuador cifras. Obtenido de http://www.ecuadorencifras.gob.ec/documentos/webinec/Estadisticas_Sociales/TIC/Resultados_principales_140515.Tic.pdf

Moya, E. J. (2016). El analfabetismo digital en docentes limita la utilización de los EVEA. Revista Publicando, 24-36.

Moya, E. J. (2016). Utopía o realidad de aplicaciones informáticas en la educación. Caso Universidad Ecuatoriana. Revista Publicando, 119-137.

Pérez-Fabara, M. A.-A.-A. (2017). Las tecnologías en el mejoramiento de los procesos educativos en la Educación Superior en América Latina. Revista Publicando, 704718.

UIT. (Mayo de 2015). Comprometida para conectar al mundo. Obtenido de https://www.itu.int/net/pressoffice/press_releases/2015/17-es.aspx 
UNESCO.

(2016).

Educación.

Obtenido

de

http://www.unesco.org/new/es/education/themes/strengthening-educationsystems/higher-education/

Vessuri, H. M. (1993). Desafíos de la educación superior en relación con la formación y la investigación ante los procesos económicos actuales y los nuevos desarrollos tecnológicos. Revista Iberoamericana de Educación, http://www.rieoei.org/oeivirt/rie02a06.htm.

Zangara, A. (2008). Puertas abiertas. Obtenido de http://www.puertasabiertas.fahce.unlp.edu.ar/numeros/n5/zangara 


\section{PARA CITAR EL ARTÍCULO INDEXADO.}

Oña Gamboa, V., Miniguano Miniguano, D., Camacho Reina, G. A., \& Naranjo Villota, D. A. (2019). La influencia de las nuevas tecnologías de la información y comunicación en la educación superior. Ciencia Digital, 3(4), 71-81. https://doi.org/10.33262/cienciadigital.v3i4.937

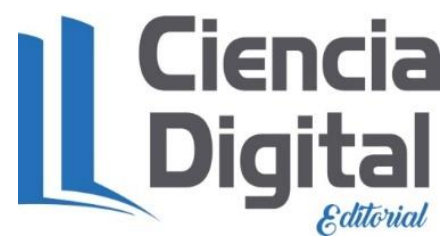

El artículo que se publica es de exclusiva responsabilidad de los autores y no necesariamente reflejan el pensamiento de la Revista Ciencia Digital.

El artículo queda en propiedad de la revista y, por tanto, su publicación parcial y/o total en otro medio tiene que ser autorizado por el director de la Revista Ciencia Digital.
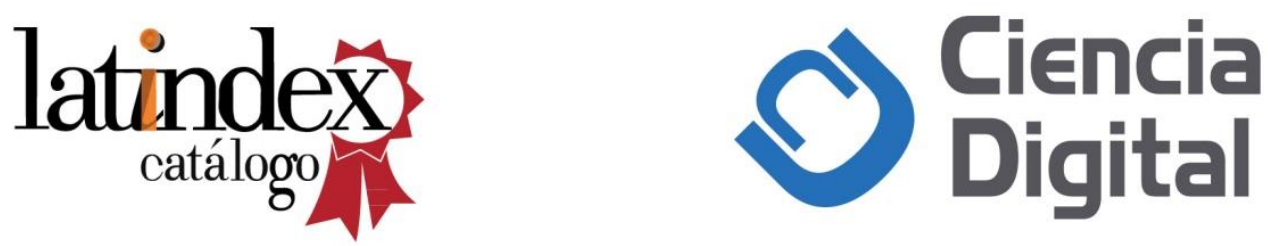